\title{
Green Synthesis of Silver Nanoparticles Using Tabebuia Aurea Leaf Extract
}

\author{
B.S Prathibha ${ }^{1}$, K. Shanmuga Priya ${ }^{2}$ \\ I (Department of Chemistry, BNMIT, Bangalore, VTU, Belgaum, Karnataka, India). \\ ${ }_{2}^{2}$ (Department of Chemistry, BNMIT, Bangalore, VTU, Belgaum, Karnataka, India).
}

\begin{abstract}
Silver nano particles were synthesized using Tabebuia aurea (TA) leaf extracts as reducing agents and silver nitrate as precursor. This method provides an economic and eco-friendly green synthesis of silver nano particles. Formation of silver nano particles were confirmed by colour change from light brown to dark brown. The aqueous silver ions when exposed to leaf broth were reduced and resulted in the green synthesis of silver nano particles. The bio reduced silver nano particles were characterized by UV-Vis spectrophotometer, SEM and EDX. The absorbance spectrum of aqueous medium containing SNPs showed a peak at around 410$440 \mathrm{~nm}$.
\end{abstract}

Keywords: Silver nano particles, Tabebuia aurea, EDX, Plant extract, SEM

\section{Introduction}

The field of nanotechnology is one of the most active areas of research in modern material sciences. Nano particles exhibit completely new or improved properties based on specific characteristics such as size, distribution and morphology. Green synthesis of metal nanoparticles (NPs) using plant extract has received greater attention due to increasing need to develop environmental sustainable technologies in nanomaterial production. Silver nanoparticles have become the focus of extensive research due to its good antimicrobial efficacy against multidrug resistant bacteria, viruses and other eukaryotic microorganisms. Nanoparticles can be synthesized using various approaches including chemical, physical, and Biological methods. Silver nanoparticles synthesized by chemical methods are toxic to different normal cells and lead to non-eco-friendly by-products which may disturb the normal cells. Ag NPs were found to exert strong acute toxic effects to various cultured cells. So, an ever increasing need for environmentally friendly, non-toxic protocols for nanoparticle synthesis leads to the developing interest in biological approaches which are free from the use of toxic chemicals as by-products. Due to the growing needs of eco-friendly nanoparticles, green methods are used for the synthesis of various metal nanoparticles.

But recently, plant extract mediated nanoparticles fabrication proved as an advantageous way over other methods. Plant extracts mediated synthesis of nanoparticles is gaining importance due to its simplicity and eco-friendliness. Silver nanoparticles are well known as one of the most universal antimicrobial substances in the field of biology and medicine due to their strong biocidal effect against microbial species, which has been used for centuries to prevent and treat various diseases, most notably infections. Silver nanoparticles also reported to possess anti-fungal, anti-inflammatory, anti-viral, anti-angiogenesis and anti-platelet activity. Recently, the development of silver nanoparticles is expanding. They are now used as part of clothing, food containers, wound dressings, ointments, and implant coatings ${ }^{1}$.

Silver nanoparticles act as biomarker in detection of early diagnosis and therapy monitoring such as the detection of tumor for cancer treatment and early diagnosis for Alzheimer's disease ${ }^{2}$. Synthesis of AgNps were adopted by many researchers using plant extracts of Aloe vera ${ }^{3}$, bamboo leaves ${ }^{4}$, Neem and triphala leaves ${ }^{5}$, Terminalia arjuna ${ }^{6}$, Camellia Sinensis ${ }^{7}$ etc.. Tabebuia aurea is used in the treatment of bronchitis, viral diseases, anti-inflammatory agent and stem bark is used for treatment of cancer. The alcohol extracts showed potent antinociceptive and anti- inflammatory activity compare to aqueous extract. Phytochemical analysis of aqueous extract showed the presence of carbohydrates, alkaloids, glycosides, tannins, saponins, phytosterols, phenolic compounds, proteins, amino acids, flavonoids, gums and mucilage ${ }^{8}$. In this article, we have synthesised AgNPs with a simple, non-toxic, cost-effective and eco-friendly method using Tabebuia aurea leaf extract which is abundantly available and not been studied for the synthesis of AgNPs. Tabebuia aurea is a species of Tabebuia native to South America in Suriname, Brazil, eastern Bolivia, Peru, Paraguay, and northern Argentina. It is a small dry season-deciduous tree growing to $8 \mathrm{~m}$ tall. The leaves are palmately compound, with five or seven leaflets, each leaflet 6-18 cm long, green with silvery scales both above and below. 


\section{A. Preparation of leaf extract:}

\section{Experimental}

TA leaves (Fig.1) were collected from a local park, washed, dried and crushed. 5\% Leaf extract was prepared by boiling $5 \mathrm{~g}$ of leaves in $100 \mathrm{ml}$ of distilled water for 1 hour. The extract was filtered using filter paper, and the filtrate was taken as stock solution. Freshly prepared aqueous extract was used for the synthesis.

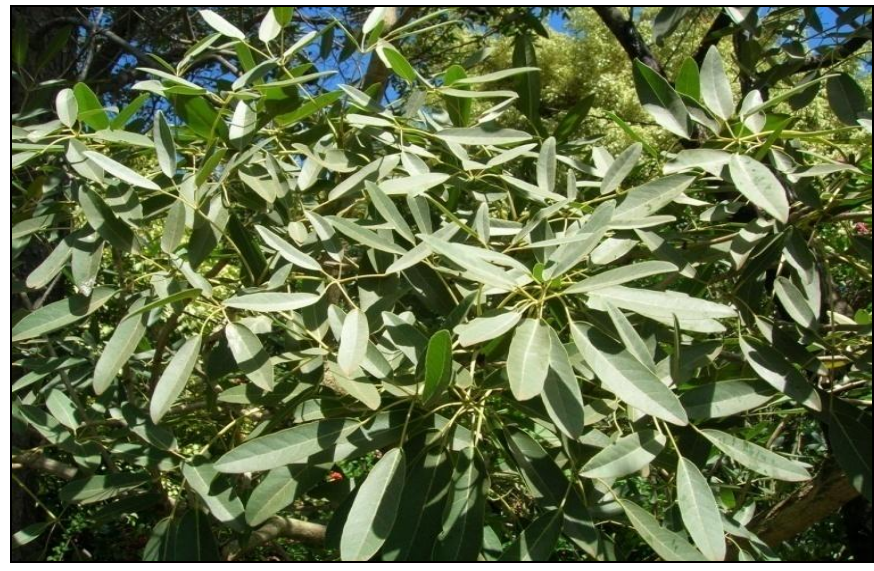

Fig.1: Tabebuia aurea Leaves

\section{B. Synthesis of Silver Nano particles:}

Silver nitrate of AR grade was purchased from a local vendor and $1 \mathrm{mM}$ solution of silver nitrate was prepared by dissolving $0.1699 \mathrm{~g}$ of silver nitrate in $1 \mathrm{~L}$ of distilled water. $10 \mathrm{ml}$ of silver nitrate solution was taken in a beaker and $1 \mathrm{ml}$ of TA extract was added drop wise with constant stirring and the reaction mixture was heated at a temperature of $80^{\circ} \mathrm{C}$ for 10 minutes. After, 15 minutes the solution turns yellow to yellow-red or dark brown indicating the formation of silver nanoparticles as shown in Fig.2. This was kept aside for 2 hours for cooling. The same procedure was repeated with different concentrations of silver nitrate keeping the volume of plant extract constant as shown in the Table [1].

Table 1: Notation of silver nanoparticles synthesized using Tabebuia aurea leaf extract.

\begin{tabular}{|l|l|l|}
\hline Sample & Plant extract $(\mathrm{ml})$ & $\mathrm{AgNO}_{3}$ Solution(ml) \\
\hline A & 1 & 10 \\
\hline B & 1 & 20 \\
\hline C & 1 & 30 \\
\hline D & 1 & 40 \\
\hline E & 1 & 50 \\
\hline F & 1 & 60 \\
\hline
\end{tabular}

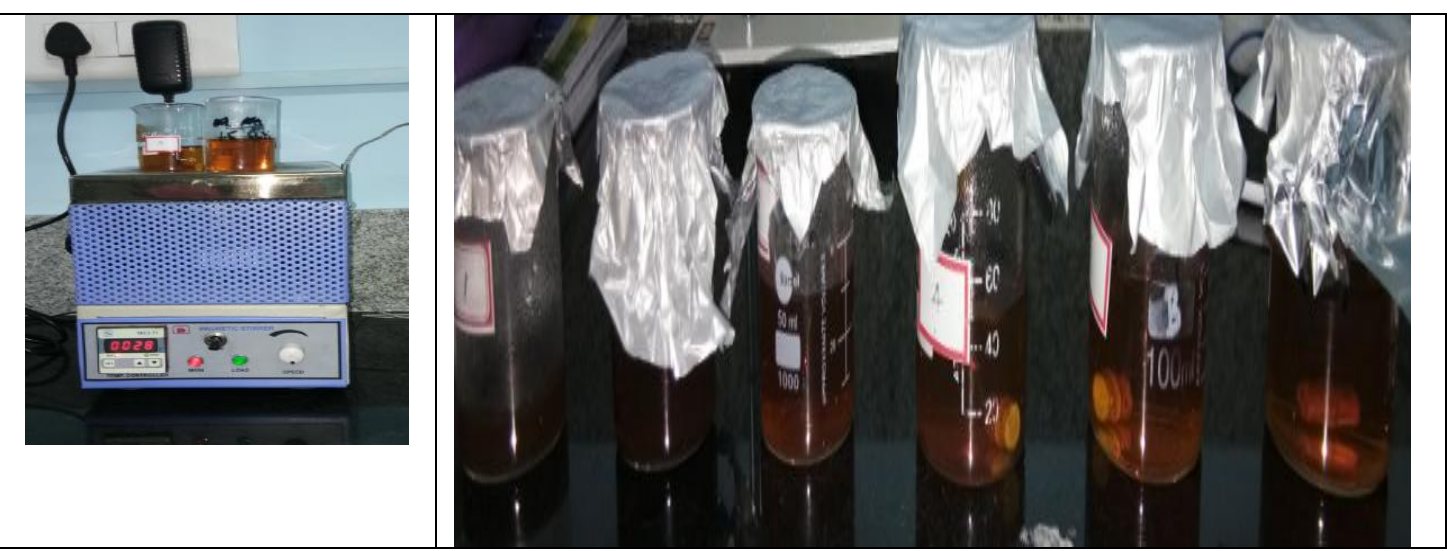

Fig 2: Experimental setup during formation of silver nano particles

\section{Characterization of Silver Nanoparticles:}

Silver nanoparticles synthesized from the Tabebuia aurea leaf extract was characterized by UVVisible spectrophotometer (UV-1601PC, SHIMADZU Make). The surface Plasmon resonance for synthesised AgNps was measured by double beam UV-Visible spectrophotometer in the wavelength range $350-700 \mathrm{~nm}$. All samples were diluted with distilled water and UV-Visible spectra were measured by taking Deionised water as 
blank solution. Scanning electron microscopic (SEM) analysis was done using SEM machine. Thin films of synthesized and stabilized silver nanoparticles were prepared on a carbon coated copper grid by just dropping a very small amount of the sample on the grid and sample was analyzed for morphology and size of the silver nanoparticles. EDX analysis gives qualitative as well as quantitative status of elements that may be involved in formation of nanoparticles.

\section{Results And Discussion}

Silver nano particles have been biosynthesized using Tabebuia aurea leaf extract, which acts as both reducing and stabilizing agent. Fig.3 represents the UV-Vis spectra of aqueous extract with different concentration of silver nitrate solution.

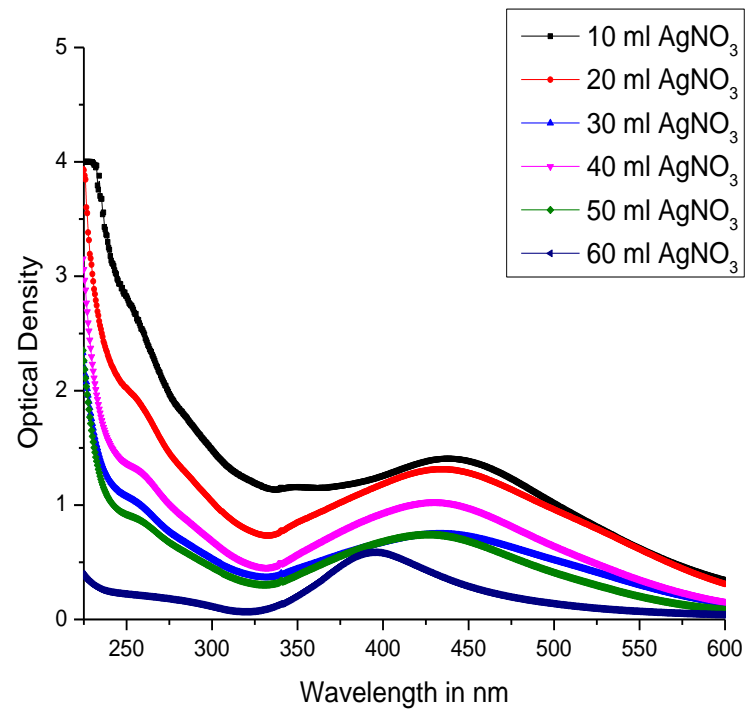

Fig 3: UV-Vis spectra of aqueous extract of TA with different concentration of $\mathrm{AgNO}_{3}$ solution.

Free electrons present in metal nano particles gives SPR (Surface Plasmon Resonance) absorption band as a result of combined vibration of electrons of metal nano particles in resonance with light wave ${ }^{9}$. Appearance of ash yellow colour indicates the formation of silver nano particles, which was further confirmed, by the appearance of absorption peak at $410-440 \mathrm{~nm}$, measured by UV visible spectroscopy ${ }^{10-11}$. The absorption peak in the above mentioned wavelength range suggests formation of spherical silver nano particles.

Colour variation from no colour to reddish brown proved the excitation of the surface Plasmon resonance in the metal nano particles. UV-Vis Spectroscopy could be used to examine size of nano particles in aqueous solutions ${ }^{12}$. Average particle size of the nano particle was calculated using following equation for effective mass approximation (Eq. 1). This equation describes the particle size in radius as a function of peak absorbance wave length for $\mathrm{AgNps}^{13}$.

$$
r(\mathrm{~nm})=\frac{-0.2963+\left(-40.1970+\frac{18620}{\lambda p}\right)}{-7.34+\frac{2418.6}{\lambda p}}
$$

The calculated size is found to be $27 \mathrm{~nm}$ this is in agreement with the SEM.

Biosynthesised silver nano particles using TA leaf extract was further confirmed using the scanning electron microscope and EDX. The SEM images in Fig. 4 showed the high density silver nano particles synthesised by TA leaf extract. It was observed from the SEM images that as the concentration of silver nitrate were increased from $10 \mathrm{ml}$ to $60 \mathrm{ml}$ the size of the nano particles also increased may be due to the aggregation of smaller particles. According to SEM analysis the silver nanoparticles were spherical in shape with varying particle size in $\mathrm{nm}$. SEM analysis further showed the particle size between 21-39 nm. Biosynthesis of silver nanoparticles are highly toxic to bacteria and hence has a very high potential in biomedical. 


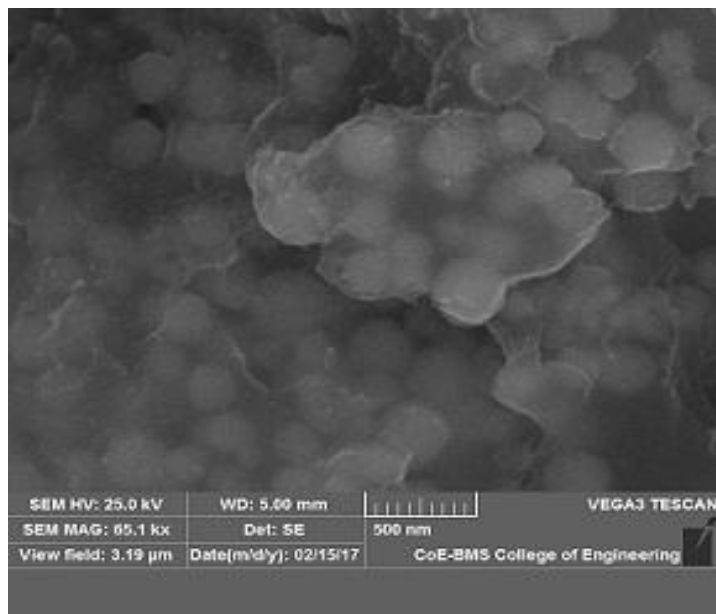

A

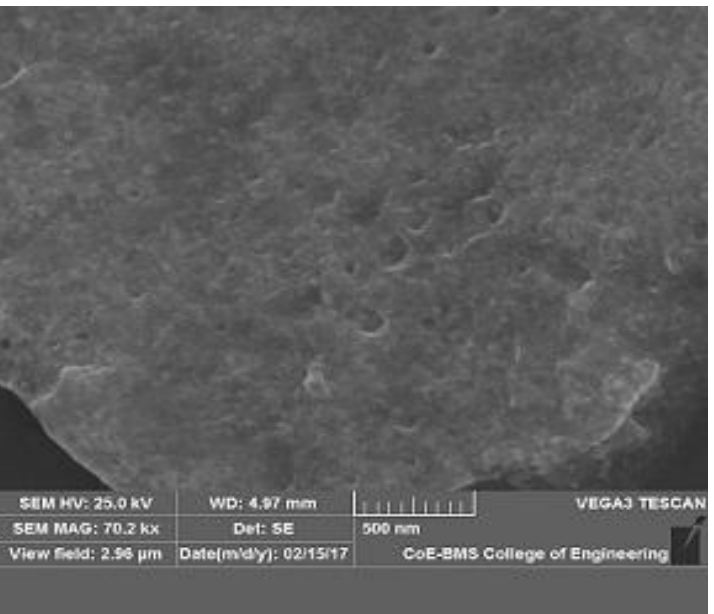

C

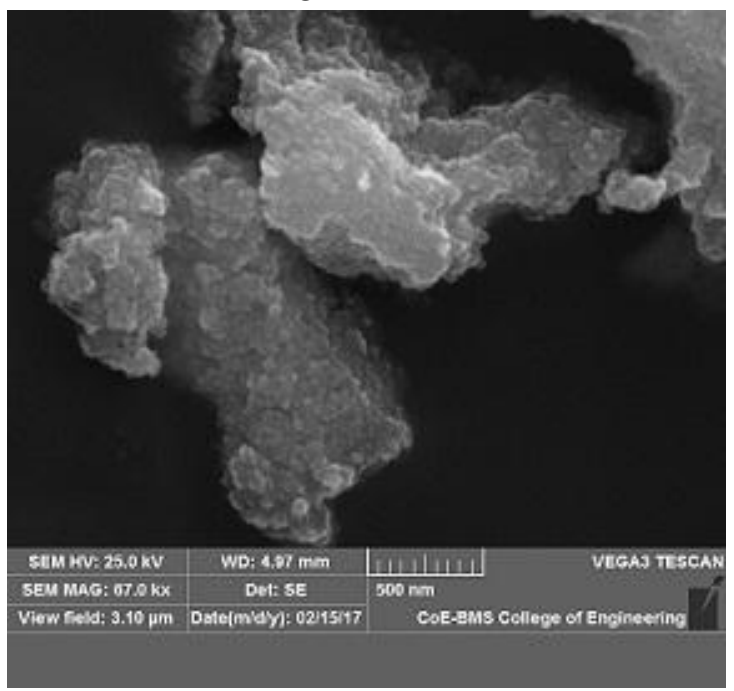

$E$

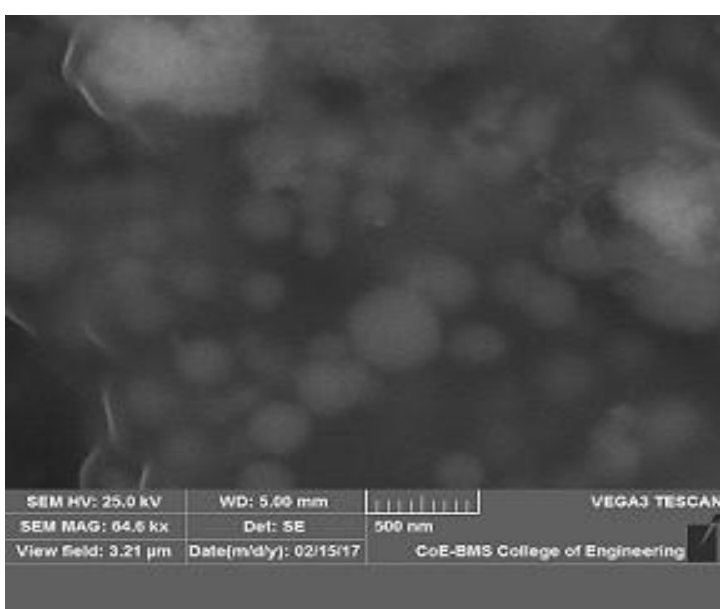

B

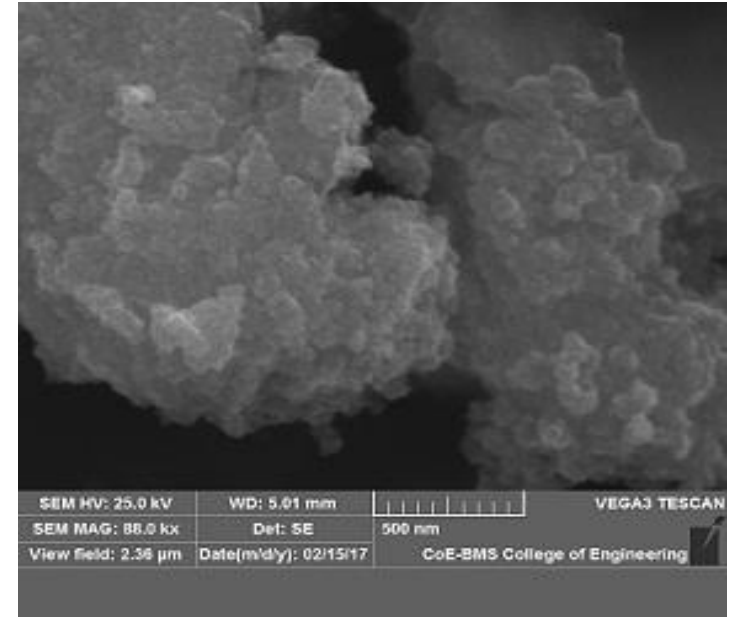

D

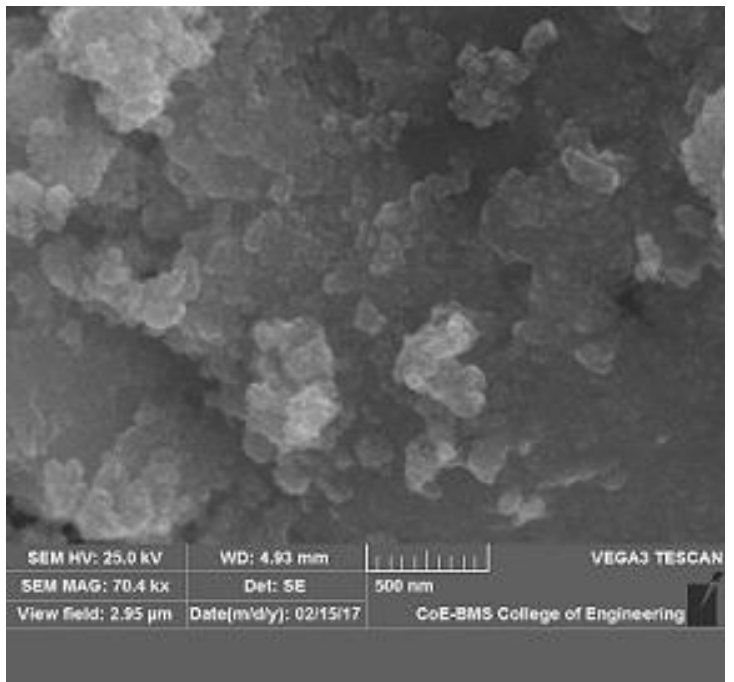

$\mathrm{F}$

Fig 4: SEM Images of Different concentrations of silver nitrate solutions (A) $10 \mathrm{ml}$ silver nitrate solution (B) $20 \mathrm{ml}$ silver nitrate solution (C) $30 \mathrm{ml}$ silver nitrate solution (D) $40 \mathrm{ml}$ silver nitrate solution (E) $50 \mathrm{ml}$ silver nitrate solution $(\mathrm{F}) 60 \mathrm{ml}$ silver nitrate solution 


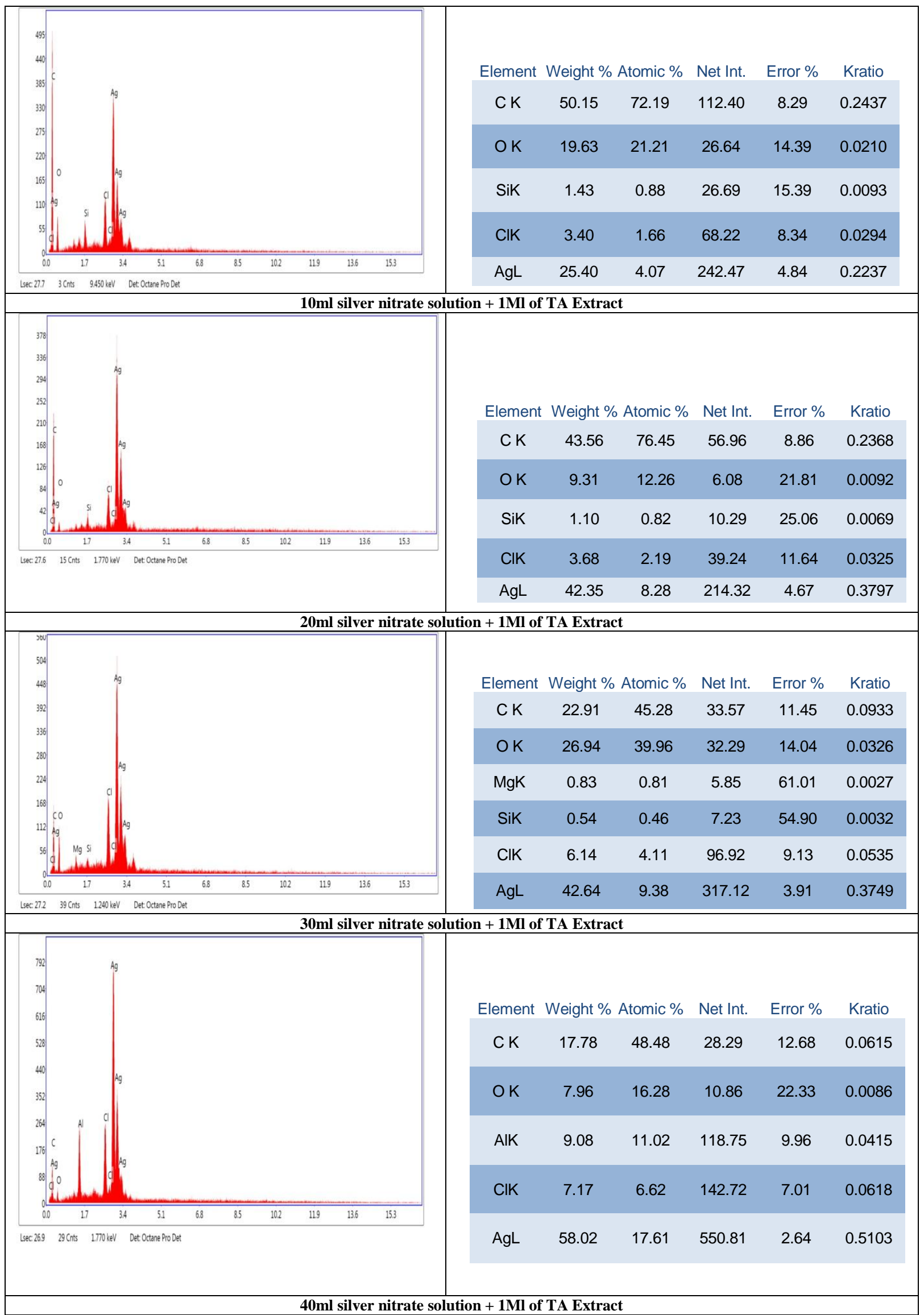




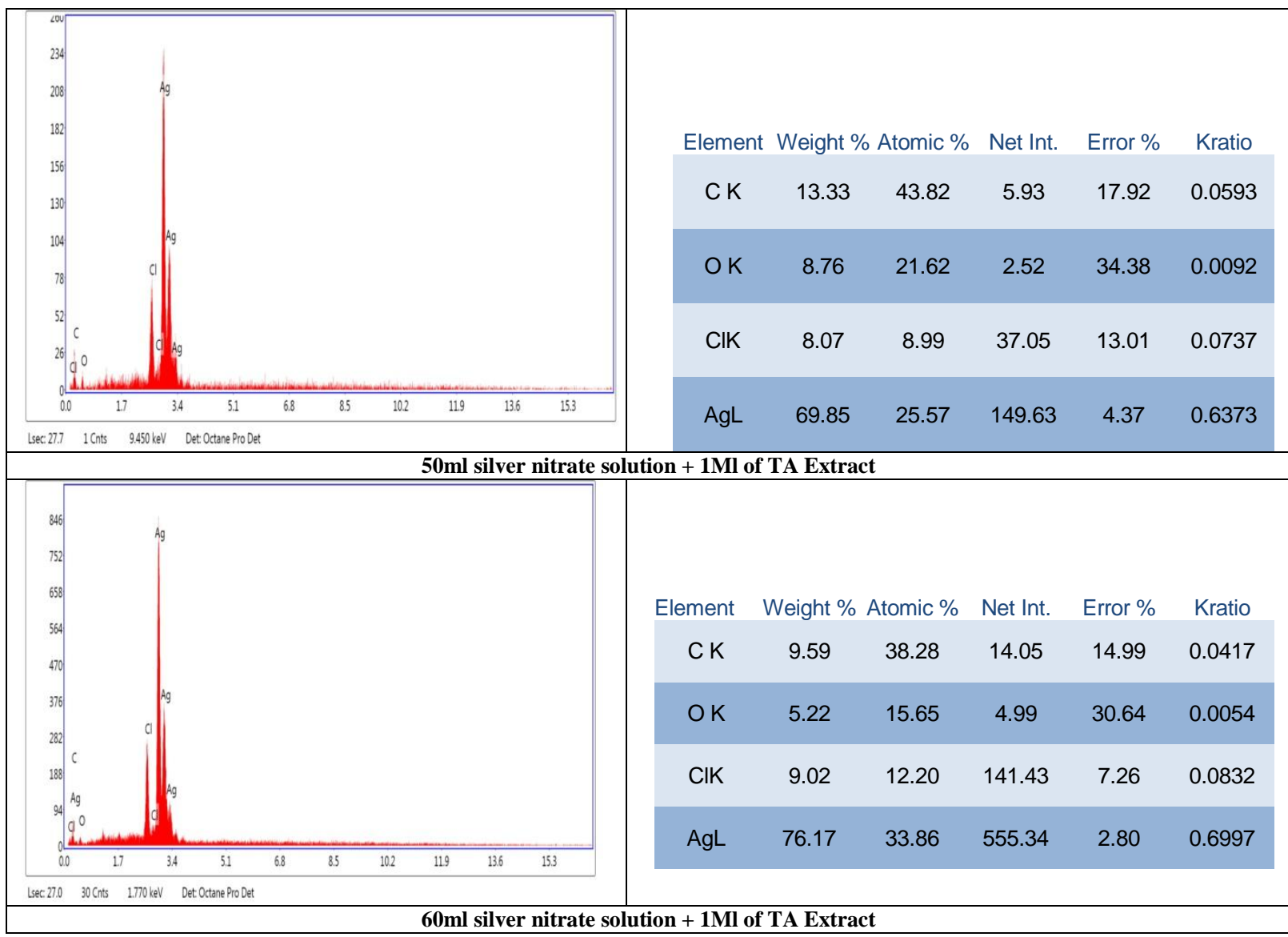

Fig. 5: EDX of Different concentrations of silver nitrate solutions

From the EDX values depicted in Fig. 5 it is clear that as the concentration of silver nitrate was increased from $10 \mathrm{ml}$ to $60 \mathrm{ml}$ there was a progressive increase in intensities of AgL spectral lines. The Ag spectra lines become more intense in films obtained from solutions with higher $\mathrm{Ag}^{+}$concentration. Literature studies explains that intensities of any spectral lines in EDX analysis are proportional to elemental abundance and EDX method does not distinguish between elemental $\mathrm{Ag}$ and $\mathrm{Ag}$ atoms in other compounds. Hence the result obtained suggests increase of $\mathrm{Ag}$ concentration in nanofilms which is confirmed by increased intensity of Ag line.

\section{Conclusion}

Silver nanoparticles were synthesized by Tabebuia aurea leaves extract.This is the first paper on the synthesis of silver nanoparticles using Tabebuia aurea leaves. The spectroscopic characterization from UVvisible, SEM, and EDX supports the stability of the biosynthesized nanoparticles. We believe that the silver nanoparticle has great potential applications in catalysis, biomedical, and pharmaceutical industries.

\section{References}

[1] Yuet Ying Loo, Buong Woei Chieng, Mitsuaki Nishibuchi and Son Radu, " Synthesis of silver nanoparticles by using tea leaf extract from Camellia Sinensis,” Inter. J. of Nanomedicine, Vol.7, (2012), 4263-4267.

[2] Singh M, Singh S, Prasad S, Gambhir IS, "Nanotechnology in Medicine and Antibacterial Effect of Silver Nanoparticles," Digest Journal of Nanomaterials and Biostructures, Vol. 3, No. 3 (2008), 115-122.

[3] Patcharaporn Tippayawat, Nutthakritta Phromviyo, Parichart Boueroy and Apiwat Chompoosor, "Green synthesis of silver nanoparticles in aloe vera plant extract prepared by a hydrothermal method and their synergistic antibacterial activity" PeerJ 4:e2589; DOI 10.7717/peerj.2589 (2016).

[4] Sohail Yasin, Lin Liu, Juming Yao "Biosynthesis of Silver Nanoparticles by Bamboo Leaves Extract and Their Antimicrobial Activity" Journal of Fiber Bioengineering and Informatics, No. 6, Vol.1 (2013), 77-84.

[5] Asmita J. Gavhane1 P. Padmanabhan, suresh P. Kamble and suresh N. Jangle, "Synthesis of silver nanoparticles using extract of Neem leaf and Triphala and evaluation of their Antimicrobial activities," Int. J. Pharma and Bio Sci., 3(3) (2012), 88-100.

[6] Shakeel Ahmed and Saiqa Ikram, "Silver Nanoparticles: One Pot Green Synthesis Using Terminalia arjuna Extract for Biological Application," J. Nanomedicine \& Nanotechnology, 6(4), (2015).

[7] Yuet Ying Loo, Buong Woei Chieng, Mitsuaki Nishibuchi, Son Radu, "Synthesis of silver nanoparticles by using tea leaf extract from Camellia Sinensis,” International Journal of Nanomedicine, 7, (2012), 4263-4267. 
[8] P. Udaya Chandrika*, A. Srinivas Rao , M. Sri Rama Chandra , NVBLA. Baby Kambampati, “Antinociceptive and AntiInflammatory Activity of Tabebeuia Aurea Leaf Extracts," Int. J. of Ayurvedic and Herbal Medicine, Vol.4, No.4, (2014), 15201526.

[9] S. S. Shankar, A. Rai, B. Ankamwar, A. Singh, A. Ahmad, M. Sastry Nat. Mater. 3, (2004), 482

[10] Krpetic Z, Scarı G, Caneva C, Speranza G, Porta F., "Gold Nanoparticles Prepared Using CapeAloe Active Components," Langmuir. 2009; 25(13): 7217-21.

[11] Shrivastava S, Bera T, Roy A, Singh G, Ramachandrarao P, Dash D. "Characterization of enhanced antibacterial effects of novel silver nanoparticles," Nanotechnology. 2007; 18: 225103

[12] B. J. Wiley, S. H. Im, J. McLellan, A. Siekkinen. Y. Xia J. Phys. Chem. B 110, (2006) 15666.

[13] K.T. Vadiraj, Shiddappa L., Belagali, "Synthesis and optical characterization of nickel doped zinc sulphide without capping agent," J Mater Sci: Mater Electron (2016) 27:2885-2889 